\title{
A Fixed Point Approach to the Stability of an Additive-Quadratic-Cubic-Quartic Functional Equation
}

\author{
Jung Rye Lee, ${ }^{1}$ Ji-hye Kim, ${ }^{2}$ and Choonkil Park ${ }^{2}$ \\ ${ }^{1}$ Department of Mathematics, Daejin University, Kyeonggi 487-711, South Korea \\ 2 Department of Mathematics, Research Institute for Natural Sciences, Hanyang University, \\ Seoul 133-791, South Korea
}

Correspondence should be addressed to Choonkil Park, baak@hanyang.ac.kr

Received 24 August 2009; Revised 16 November 2009; Accepted 10 January 2010

Academic Editor: Fabio Zanolin

Copyright (C) 2010 Jung Rye Lee et al. This is an open access article distributed under the Creative Commons Attribution License, which permits unrestricted use, distribution, and reproduction in any medium, provided the original work is properly cited.

Using the fixed point method, we prove the generalized Hyers-Ulam stability of the following additive-quadratic-cubic-quartic functional equation $f(x+2 y)+f(x-2 y)=4 f(x+y)+4 f(x-y)-$ $6 f(x)+f(2 y)+f(-2 y)-4 f(y)-4 f(-y)$ in Banach spaces.

\section{Introduction and Preliminaries}

The stability problem of functional equations is originated from a question of Ulam [1] concerning the stability of group homomorphisms. Hyers [2] gave a first affirmative partial answer to the question of Ulam for Banach spaces. Hyers' Theorem was generalized by Aoki [3] for additive mappings and by Th. M. Rassias [4] for linear mappings by considering an unbounded Cauchy difference. The paper of Th. M. Rassias [4] has provided a lot of influence in the development of what we call generalized Hyers-Ulam stability or as Hyers-Ulam-Rassias stability of functional equations. A generalization of the Th. M. Rassias theorem was obtained by Găvruţa [5] by replacing the unbounded Cauchy difference by a general control function in the spirit of Th. M. Rassias' approach.

The functional equation

$$
f(x+y)+f(x-y)=2 f(x)+2 f(y)
$$


is called a quadratic functional equation. In particular, every solution of the quadratic functional equation is said to be a quadratic mapping. A generalized Hyers-Ulam stability problem for the quadratic functional equation was proved by Skof [6] for mappings $f: X \rightarrow Y$, where $X$ is a normed space and $Y$ is a Banach space. Cholewa [7] noticed that the theorem of Skof is still true if the relevant domain $X$ is replaced by an Abelian group. Czerwik [8] proved the generalized Hyers-Ulam stability of the quadratic functional equation. The stability problems of several functional equations have been extensively investigated by a number of authors, and there are many interesting results concerning this problem (see [9-19]).

In [20], Jun and Kim considered the following cubic functional equation

$$
f(2 x+y)+f(2 x-y)=2 f(x+y)+2 f(x-y)+12 f(x),
$$

which is called a cubic functional equation, and every solution of the cubic functional equation is said to be a cubic mapping.

In [21], Lee et al. considered the following quartic functional equation

$$
f(2 x+y)+f(2 x-y)=4 f(x+y)+4 f(x-y)+24 f(x)-6 f(y)
$$

which is called a quartic functional equation and every solution of the quartic functional equation is said to be a quartic mapping. Quartic functional equations have been investigated in $[22,23]$. satisfies

Let $X$ be a set. A function $d: X \times X \rightarrow[0, \infty]$ is called a generalized metric on $X$ if $d$

(1) $d(x, y)=0$ if and only if $x=y$;

(2) $d(x, y)=d(y, x)$ for all $x, y \in X$;

(3) $d(x, z) \leq d(x, y)+d(y, z)$ for all $x, y, z \in X$.

We recall a fundamental result in fixed point theory.

Theorem 1.1 (see $[24,25])$. Let $(X, d)$ be a complete generalized metric space and let $J: X \rightarrow X$ be a strictly contractive mapping with Lipschitz constant $L<1$. Then for each given element $x \in X$, either

$$
d\left(J^{n} x, J^{n+1} x\right)=\infty
$$

for all nonnegative integers $n$ or there exists a positive integer $n_{0}$ such that

(1) $d\left(J^{n} x, J^{n+1} x\right)<\infty$, for all $n \geq n_{0}$;

(2) the sequence $\left\{J^{n} x\right\}$ converges to a fixed point $y^{*}$ of $J$;

(3) $y^{*}$ is the unique fixed point of $J$ in the set $Y=\left\{y \in X \mid d\left(J^{n_{0}} x, y\right)<\infty\right\}$;

(4) $d\left(y, y^{*}\right) \leq(1 /(1-L)) d(y, J y)$ for all $y \in Y$.

In 1996, Isac and Th. M. Rassias [26] were the first to provide applications of stability theory of functional equations for the proof of new fixed point theorems with applications. By using fixed point methods, the stability problems of several functional equations have been extensively investigated by a number of authors (see [27-32]). 
This paper is organized as follows. In Section 2, we prove the generalized Hyers-Ulam stability of the additive-quadratic-cubic-quartic functional equation

$$
f(x+2 y)+f(x-2 y)=4 f(x+y)+4 f(x-y)-6 f(x)+f(2 y)+f(-2 y)-4 f(y)-4 f(-y)
$$

in Banach spaces for an odd case. In Section 3, we prove the generalized Hyers-Ulam stability of the additive-quadratic-cubic-quartic functional equation (1.5) in Banach spaces for an even case.

Throughout this paper, assume that $X$ is a vector space and that $Y$ is a Banach space.

\section{Generalized Hyers-Ulam Stability of the Functional Equation (1.5): An Odd Case}

For a given mapping $f: X \rightarrow Y$, we define

$$
\begin{aligned}
D f(x, y):= & f(x+2 y)+f(x-2 y)-4 f(x+y)-4 f(x-y)+6 f(x) \\
& -f(2 y)-f(-2 y)+4 f(y)+4 f(-y)
\end{aligned}
$$

for all $x, y \in X$.

Using the fixed point method, we prove the generalized Hyers-Ulam stability of the functional equation $D f(x, y)=0$ in Banach spaces: an odd case.

Note that the fundamental ideas in the proofs of the main results in Sections 2 and 3 are contained in $[24,27,28]$.

Theorem 2.1. Let $\varphi: X^{2} \rightarrow[0, \infty)$ be a function such that there exists an $L<1$ with

$$
\varphi(x, y) \leq \frac{L}{8} \varphi(2 x, 2 y)
$$

for all $x, y \in X$. Let $f: X \rightarrow Y$ be an odd mapping satisfying

$$
\|D f(x, y)\| \leq \varphi(x, y)
$$

for all $x, y \in X$. Then there is a unique cubic mapping $C: X \rightarrow Y$ such that

$$
\|f(2 x)-2 f(x)-C(x)\| \leq \frac{L}{8-8 L}(4 \varphi(x, x)+\varphi(2 x, x))
$$

for all $x \in X$.

Proof. Letting $x=y$ in (2.3), we get

$$
\|f(3 y)-4 f(2 y)+5 f(y)\| \leq \varphi(y, y)
$$

for all $y \in X$. 
Replacing $x$ by $2 y$ in (2.3), we get

$$
\|f(4 y)-4 f(3 y)+6 f(2 y)-4 f(y)\| \leq \varphi(2 y, y)
$$

for all $y \in X$.

By (2.5) and (2.6),

$$
\begin{aligned}
\| f(4 y) & -10 f(2 y)+16 f(y) \| \\
\leq & \|4(f(3 y)-4 f(2 y)+5 f(y))\| \\
& +\|f(4 y)-4 f(3 y)+6 f(2 y)-4 f(y)\| \\
\leq & 4 \varphi(y, y)+\varphi(2 y, y)
\end{aligned}
$$

for all $y \in X$. Letting $y:=x / 2$ and $g(x):=f(2 x)-2 f(x)$ for all $x \in X$, we get

$$
\left\|g(x)-8 g\left(\frac{x}{2}\right)\right\| \leq 4 \varphi\left(\frac{x}{2}, \frac{x}{2}\right)+\varphi\left(x, \frac{x}{2}\right)
$$

for all $x \in X$.

Consider the set

$$
S:=\{g: X \longrightarrow Y\}
$$

and introduce the generalized metric on $S$ :

$$
d(g, h)=\inf \left\{\mu \in \mathbb{R}_{+}:\|g(x)-h(x)\| \leq \mu(4 \varphi(x, x)+\varphi(2 x, x)), \forall x \in X\right\},
$$

where, as usual, $\inf \phi=+\infty$. It is easy to show that $(S, d)$ is complete (see the proof of Lemma 2.1 of [33]).

Now we consider the linear mapping $J: S \rightarrow S$ such that

$$
J g(x):=8 g\left(\frac{x}{2}\right)
$$

for all $x \in X$.

Let $g, h \in S$ be given such that $d(g, h)=\varepsilon$. Then

$$
\|g(x)-h(x)\| \leq 4 \varphi(x, x)+\varphi(2 x, x)
$$

for all $x \in X$. Hence

$$
\|J g(x)-J h(x)\|=\left\|8 g\left(\frac{x}{2}\right)-8 h\left(\frac{x}{2}\right)\right\| \leq L(4 \varphi(x, x)+\varphi(2 x, x))
$$


for all $x \in X$. So $d(g, h)=\varepsilon$ implies that $d(J g, J h) \leq L \varepsilon$. This means that

$$
d(J g, J h) \leq L d(g, h)
$$

for all $g, h \in S$.

It follows from (2.8) that

$$
\left\|g(x)-8 g\left(\frac{x}{2}\right)\right\| \leq \frac{L}{8}(4 \varphi(x, x)+\varphi(2 x, x))
$$

for all $x \in X$. So $d(g, J g) \leq L / 8$.

By Theorem 1.1, there exists a mapping $C: X \rightarrow Y$ satisfying the following.

(1) $C$ is a fixed point of $J$, that is,

$$
C\left(\frac{x}{2}\right)=\frac{1}{8} C(x)
$$

for all $x \in X$. Since $g: X \rightarrow Y$ is odd, $C: X \rightarrow Y$ is an odd mapping. The mapping $C$ is a unique fixed point of $J$ in the set

$$
M=\{g \in S: d(f, g)<\infty\} .
$$

This implies that $C$ is a unique mapping satisfying (2.16) such that there exists a $\mu \in(0, \infty)$ satisfying

$$
\|g(x)-C(x)\| \leq \mu(4 \varphi(x, x)+\varphi(2 x, x))
$$

for all $x \in X$.

(2) $d\left(J^{n} g, C\right) \rightarrow 0$ as $n \rightarrow \infty$. This implies the equality

$$
\lim _{n \rightarrow \infty} 8^{n} g\left(\frac{x}{2^{n}}\right)=C(x)
$$

for all $x \in X$.

(3) $d(g, C) \leq(1 /(1-L)) d(g, J g)$, which implies the inequality

$$
d(g, C) \leq \frac{L}{8-8 L}
$$

This implies that the inequality (2.4) holds.

By (2.3),

$$
\left\|8^{n} D g\left(\frac{x}{2^{n}}, \frac{y}{2^{n}}\right)\right\| \leq 8^{n}\left(\varphi\left(\frac{2 x}{2^{n}}, \frac{2 y}{2^{n}}\right)+2 \varphi\left(\frac{x}{2^{n}}, \frac{y}{2^{n}}\right)\right)
$$


for all $x, y \in X$ and all $n \in \mathbb{N}$. So

$$
\left\|8^{n} D g\left(\frac{x}{2^{n}}, \frac{y}{2^{n}}\right)\right\| \leq L^{n}(\varphi(2 x, 2 y)+2 \varphi(x, y))
$$

for all $x, y \in X$ and all $n \in \mathbb{N}$. So

$$
\|D C(x, y)\|=0
$$

for all $x, y \in X$. Thus the mapping $C: X \rightarrow Y$ is cubic, as desired.

Corollary 2.2. Let $\theta \geq 0$ and let $p$ be a real number with $p>3$. Let $X$ be a normed vector space with norm $\|\cdot\|$. Let $f: X \rightarrow Y$ be an odd mapping satisfying

$$
\|D f(x, y)\| \leq \theta\left(\|x\|^{p}+\|y\|^{p}\right)
$$

for all $x, y \in X$. Then there is a unique cubic mapping $C: X \rightarrow Y$ such that

$$
\|f(2 x)-2 f(x)-C(x)\| \leq \frac{2^{p}+9}{2^{p}-8} \theta\|x\|^{p}
$$

for all $x \in X$.

Proof. The proof follows from Theorem 2.1 by taking

$$
\varphi(x, y):=\theta\left(\|x\|^{p}+\|y\|^{p}\right)
$$

for all $x, y \in X$. Then we can choose $L=2^{3-p}$ and we get the desired result.

Theorem 2.3. Let $\varphi: X^{2} \rightarrow[0, \infty)$ be a function such that there exists an $L<1$ with

$$
\varphi(x, y) \leq 8 L \varphi\left(\frac{x}{2}, \frac{y}{2}\right)
$$

for all $x, y \in X$. Let $f: X \rightarrow Y$ be an odd mapping satisfying (2.3). Then there is a unique cubic mapping $C: X \rightarrow Y$ such that

$$
\|f(2 x)-2 f(x)-C(x)\| \leq \frac{1}{8-8 L}(4 \varphi(x, x)+\varphi(2 x, x))
$$

for all $x \in X$.

Proof. Let $(S, d)$ be the generalized metric space defined in the proof of Theorem 2.1.

Consider the linear mapping $J: S \rightarrow S$ such that

$$
J g(x):=\frac{1}{8} g(2 x)
$$

for all $x \in X$. 
Fixed Point Theory and Applications

It follows from (2.8) that

$$
\left\|g(x)-\frac{1}{8} g(2 x)\right\| \leq \frac{1}{8}(4 \varphi(x, x)+\varphi(2 x, x))
$$

for all $x \in X$. So $d(g, J g) \leq 1 / 8$.

The rest of the proof is similar to the proof of Theorem 2.1.

Corollary 2.4. Let $\theta \geq 0$ and let $p$ be a real number with $0<p<3$. Let $X$ be a normed vector space with norm $\|\cdot\|$. Let $f: X \rightarrow Y$ be an odd mapping satisfying (2.24). Then there is a unique cubic mapping $C: X \rightarrow Y$ such that

$$
\|f(2 x)-2 f(x)-C(x)\| \leq \frac{9+2^{p}}{8-2^{p}} \theta\|x\|^{p}
$$

for all $x \in X$.

Proof. The proof follows from Theorem 2.3 by taking

$$
\varphi(x, y):=\theta\left(\|x\|^{p}+\|y\|^{p}\right)
$$

for all $x, y \in X$. Then we can choose $L=2^{p-3}$ and we get the desired result.

Theorem 2.5. Let $\varphi: X^{2} \rightarrow[0, \infty)$ be a function such that there exists an $L<1$ with

$$
\varphi(x, y) \leq \frac{L}{2} \varphi(2 x, 2 y)
$$

for all $x, y \in X$. Let $f: X \rightarrow Y$ be an odd mapping satisfying (2.3). Then there is a unique additive mapping $A: X \rightarrow Y$ such that

$$
\|f(2 x)-8 f(x)-A(x)\| \leq \frac{L}{2-2 L}(4 \varphi(x, x)+\varphi(2 x, x))
$$

for all $x \in X$.

Proof. Let $(S, d)$ be the generalized metric space defined in the proof of Theorem 2.1.

Letting $y:=x / 2$ and $h(x):=f(2 x)-8 f(x)$ for all $x \in X$ in (2.7), we get

$$
\left\|h(x)-2 h\left(\frac{x}{2}\right)\right\| \leq 4 \varphi\left(\frac{x}{2}, \frac{x}{2}\right)+\varphi\left(x, \frac{x}{2}\right)
$$

for all $x \in X$.

Now we consider the linear mapping $J: S \rightarrow S$ such that

$$
J h(x):=2 h\left(\frac{x}{2}\right)
$$

for all $x \in X$. 
It follows from (2.35) that

$$
\left\|h(x)-2 h\left(\frac{x}{2}\right)\right\| \leq 2 L \varphi(x, x)+\frac{L}{2} \varphi(2 x, x)
$$

for all $x \in X$. So $d(h, J h) \leq L / 2$.

The rest of the proof is similar to the proof of Theorem 2.1.

Corollary 2.6. Let $\theta \geq 0$ and let $p$ be a real number with $p>1$. Let $X$ be a normed vector space with norm $\|\cdot\|$. Let $f: X \rightarrow Y$ be an odd mapping satisfying (2.24). Then there is a unique additive mapping $A: X \rightarrow Y$ such that

$$
\|f(2 x)-8 f(x)-A(x)\| \leq \frac{2^{p}+9}{2^{p}-2} \theta\|x\|^{p}
$$

for all $x \in X$.

Theorem 2.7. Let $\varphi: X^{2} \rightarrow[0, \infty)$ be a function such that there exists an $L<1$ with

$$
\varphi(x, y) \leq 2 L \varphi\left(\frac{x}{2}, \frac{y}{2}\right)
$$

for all $x, y \in X$. Let $f: X \rightarrow Y$ be an odd mapping satisfying (2.3). Then there is a unique additive mapping $A: X \rightarrow Y$ such that

$$
\|f(2 x)-8 f(x)-A(x)\| \leq \frac{1}{2-2 L}(4 \varphi(x, x)+\varphi(2 x, x))
$$

for all $x \in X$.

Proof. Let $(S, d)$ be the generalized metric space defined in the proof of Theorem 2.1.

Consider the linear mapping $J: S \rightarrow S$ such that

$$
J h(x):=\frac{1}{2} h(2 x)
$$

for all $x \in X$.

It follows from (2.35) that

$$
\left\|h(x)-\frac{1}{2} h(2 x)\right\| \leq 2 \varphi(x, x)+\frac{1}{2} \varphi(2 x, x)
$$

for all $x \in X$. So $d(h, J h) \leq 1 / 2$.

The rest of the proof is similar to the proof of Theorem 2.1. 
Corollary 2.8. Let $\theta \geq 0$ and let $p$ be a real number with $0<p<1$. Let $X$ be a normed vector space with norm $\|\cdot\|$. Let $f: X \rightarrow Y$ be an odd mapping satisfying (2.24). Then there is a unique additive mapping $A: X \rightarrow Y$ such that

$$
\|f(2 x)-8 f(x)-A(x)\| \leq \frac{9+2^{p}}{2-2^{p}} \theta\|x\|^{p}
$$

for all $x \in X$

\section{Generalized Hyers-Ulam Stability of the Functional Equation (1.5): An Even Case}

Using the fixed point method, we prove the generalized Hyers-Ulam stability of the functional equation $D f(x, y)=0$ in Banach spaces: an even case.

Theorem 3.1. Let $\varphi: X^{2} \rightarrow[0, \infty)$ be a function such that there exists an $L<1$ with

$$
\varphi(x, y) \leq \frac{L}{16} \varphi(2 x, 2 y)
$$

for all $x, y \in X$. Let $f: X \rightarrow Y$ be an even mapping satisfying $f(0)=0$ and (2.3). Then there is a unique quartic mapping $Q: X \rightarrow Y$ such that

$$
\|f(2 x)-4 f(x)-Q(x)\| \leq \frac{L}{16-16 L}(4 \varphi(x, x)+\varphi(2 x, x))
$$

for all $x \in X$.

Proof. Letting $x=y$ in (2.3), we get

$$
\|f(3 y)-6 f(2 y)+15 f(y)\| \leq \varphi(y, y)
$$

for all $y \in X$.

Replacing $x$ by $2 y$ in (2.3), we get

$$
\|f(4 y)-4 f(3 y)+4 f(2 y)+4 f(y)\| \leq \varphi(2 y, y)
$$

for all $y \in X$.

By (3.4) and (3.5),

$$
\begin{aligned}
\| f(4 x) & -20 f(2 x)+64 f(x) \| \\
\leq & \|4(f(3 x)-6 f(2 x)+15 f(x))\|+\|f(4 x)-4 f(3 x)+4 f(2 x)+4 f(x)\| \\
\leq & 4 \varphi(x, x)+\varphi(2 x, x)
\end{aligned}
$$


for all $x \in X$. Letting $g(x):=f(2 x)-4 f(x)$ for all $x \in X$, we get

$$
\left\|g(x)-16 g\left(\frac{x}{2}\right)\right\| \leq 4 \varphi\left(\frac{x}{2}, \frac{x}{2}\right)+\varphi\left(x, \frac{x}{2}\right)
$$

for all $x \in X$.

Let $(S, d)$ be the generalized metric space defined in the proof of Theorem 2.1.

It follows from (3.16) that

$$
\left\|g(x)-16 g\left(\frac{x}{2}\right)\right\| \leq \frac{L}{16}(4 \varphi(x, x)+\varphi(2 x, x))
$$

for all $x \in X$. So $d(g, J g) \leq L / 16$.

The rest of the proof is similar to the proof of Theorem 2.1.

Corollary 3.2. Let $\theta \geq 0$ and let $p$ be a real number with $p>4$. Let $X$ be a normed vector space with norm $\|\cdot\|$. Let $f: X \rightarrow Y$ be an even mapping satisfying $f(0)=0$ and (2.24). Then there is unique quartic mapping $Q: X \rightarrow Y$ such that

$$
\|f(2 x)-4 f(x)-Q(x)\| \leq \frac{2^{p}+9}{2^{p}-16} \theta\|x\|^{p}
$$

for all $x \in X$.

Theorem 3.3. Let $\varphi: X^{2} \rightarrow[0, \infty)$ be a function such that there exists an $L<1$ with

$$
\varphi(x, y) \leq 16 L \varphi\left(\frac{x}{2}, \frac{y}{2}\right)
$$

for all $x, y \in X$. Let $f: X \rightarrow Y$ be an even mapping satisfying $f(0)=0$ and (2.3). Then there is a unique quartic mapping $Q: X \rightarrow Y$ such that

$$
\|f(2 x)-4 f(x)-Q(x)\| \leq \frac{1}{16-16 L}(4 \varphi(x, x)+\varphi(2 x, x))
$$

for all $x \in X$.

Proof. Let $(S, d)$ be the generalized metric space defined in the proof of Theorem 2.1.

Consider the linear mapping $J: S \rightarrow S$ such that

$$
J g(x):=\frac{1}{16} g(2 x)
$$

for all $x \in X$. 
Fixed Point Theory and Applications

It follows from (3.16) that

$$
\left\|g(x)-\frac{1}{16} g(2 x)\right\| \leq \frac{1}{16}(4 \varphi(x, x)+\varphi(2 x, x))
$$

for all $x \in X$. So $d(g, J g) \leq 1 / 16$.

The rest of the proof is similar to the proof of Theorem 2.1.

Corollary 3.4. Let $\theta \geq 0$ and let $p$ be a real number with $0<p<4$. Let $X$ be a normed vector space with norm $\|\cdot\|$. Let $f: X \rightarrow Y$ be an even mapping satisfying $f(0)=0$ and (2.24). Then there is a unique quartic mapping $Q: X \rightarrow Y$ such that

$$
\|f(2 x)-4 f(x)-Q(x)\| \leq \frac{9+2^{p}}{16-2^{p}} \theta\|x\|^{p}
$$

for all $x \in X$.

Theorem 3.5. Let $\varphi: X^{2} \rightarrow[0, \infty)$ be a function such that there exists an $L<1$ with

$$
\varphi(x, y) \leq \frac{L}{4} \varphi(2 x, 2 y)
$$

for all $x, y \in X$. Let $f: X \rightarrow Y$ be an even mapping satisfying $f(0)=0$ and (2.3). Then there is a unique quadratic mapping $T: X \rightarrow Y$ such that

$$
\|f(2 x)-16 f(x)-T(x)\| \leq \frac{L}{4-4 L}(4 \varphi(x, x)+\varphi(2 x, x))
$$

for all $x \in X$.

Proof. Let $(S, d)$ be the generalized metric space defined in the proof of Theorem 2.1.

Letting $h(x):=f(2 x)-16 f(x)$ for all $x \in X$ in (3.6), we get

$$
\left\|h(x)-4 h\left(\frac{x}{2}\right)\right\| \leq 4 \varphi\left(\frac{x}{2}, \frac{x}{2}\right)+\varphi\left(x, \frac{x}{2}\right)
$$

for all $x \in X$.

Now we consider the linear mapping $J: S \rightarrow S$ such that

$$
\operatorname{Jh}(x):=4 h\left(\frac{x}{2}\right)
$$

for all $x \in X$. 
It follows from (3.16)that

$$
\left\|h(x)-4 h\left(\frac{x}{2}\right)\right\| \leq L \varphi(x, x)+\frac{L}{4} \varphi(2 x, x)
$$

for all $x \in X$. So $d(h, J h) \leq L / 4$.

The rest of the proof is similar to the proof of Theorem 2.1.

Corollary 3.6. Let $\theta \geq 0$ and let $p$ be a real number with $p>2$. Let $X$ be a normed vector space with norm $\|\cdot\|$. Let $f: X \rightarrow Y$ be an even mapping satisfying $f(0)=0$ and (2.24). Then there is a unique quadratic mapping $T: X \rightarrow Y$ such that

$$
\|f(2 x)-16 f(x)-T(x)\| \leq \frac{2^{p}+9}{2^{p}-4} \theta\|x\|^{p}
$$

for all $x \in X$.

Theorem 3.7. Let $\varphi: X^{2} \rightarrow[0, \infty)$ be a function such that there exists an $L<1$ with

$$
\varphi(x, y) \leq 4 L \varphi\left(\frac{x}{2}, \frac{y}{2}\right)
$$

for all $x, y \in X$. Let $f: X \rightarrow Y$ be an even mapping satisfying $f(0)=0$ and (2.3). Then there is a unique quadratic mapping $T: X \rightarrow Y$ such that

$$
\|f(2 x)-16 f(x)-T(x)\| \leq \frac{1}{4-4 L}(4 \varphi(x, x)+\varphi(2 x, x))
$$

for all $x \in X$.

Proof. Let $(S, d)$ be the generalized metric space defined in the proof of Theorem 2.1.

Consider the linear mapping $J: S \rightarrow S$ such that

$$
J h(x):=\frac{1}{4} h(2 x)
$$

for all $x \in X$.

It follows from (3.16) that

$$
\left\|h(x)-\frac{1}{4} h(2 x)\right\| \leq \varphi(x, x)+\frac{1}{4} \varphi(2 x, x)
$$

for all $x \in X$. So $d(h, J h) \leq 1 / 4$.

The rest of the proof is similar to the proof of Theorem 2.1. 
Corollary 3.8. Let $\theta \geq 0$ and let $p$ be a real number with $0<p<2$. Let $X$ be a normed vector space with norm $\|\cdot\|$. Let $f: X \rightarrow Y$ be an even mapping satisfying $f(0)=0$ and (2.24). Then there is a unique quadratic mapping $T: X \rightarrow Y$ such that

$$
\|f(2 x)-16 f(x)-T(x)\| \leq \frac{9+2^{p}}{4-2^{p}} \theta\|x\|^{p}
$$

for all $x \in X$.

\section{Generalized Hyers-Ulam Stability of the Functional Equation (1.5)}

One can easily show that an odd mapping $f: X \rightarrow Y$ satisfies (1.5) if and only if the odd mapping $f: X \rightarrow Y$ is an additive-cubic mapping, that is,

$$
f(x+2 y)+f(x-2 y)=4 f(x+y)+4 f(x-y)-6 f(x) .
$$

It was shown in of [34, Lemma 2.2] that $g(x):=f(2 x)-2 f(x)$ and $h(x):=f(2 x)-8 f(x)$ are cubic and additive, respectively, and that $f(x)=(1 / 6) g(x)-(1 / 6) h(x)$.

One can easily show that an even mapping $f: X \rightarrow Y$ satisfies (1.5) if and only if the even mapping $f: X \rightarrow Y$ is a quadratic-quartic mapping, that is,

$$
f(x+2 y)+f(x-2 y)=4 f(x+y)+4 f(x-y)-6 f(x)+2 f(2 y)-8 f(y) .
$$

It was shown in of [35, Lemma 2.1] that $g(x):=f(2 x)-4 f(x)$ and $h(x):=f(2 x)-16 f(x)$ are quartic and quadratic, respectively, and that $f(x)=(1 / 12) g(x)-(1 / 12) h(x)$. Functional equations of mixed type have been investigated in [36, 37].

Let $f_{o}(x):=(f(x)-f(-x)) / 2$ and $f_{e}(x):=(f(x)+f(-x)) / 2$. Then $f_{o}$ is odd and $f_{e}$ is even. $f_{o}$ and $f_{e}$ satisfy the functional equation (1.5). Let $g_{o}(x):=f_{o}(2 x)-2 f_{o}(x)$ and $h_{o}(x):=f_{o}(2 x)-8 f_{o}(x)$. Then $f_{o}(x)=(1 / 6) g_{o}(x)-(1 / 6) h_{o}(x)$. Let $g_{e}(x):=f_{e}(2 x)-4 f_{e}(x)$ and $h_{e}(x):=f_{e}(2 x)-16 f_{e}(x)$. Then $f_{e}(x)=(1 / 12) g_{e}(x)-(1 / 12) h_{e}(x)$. Thus

$$
f(x)=\frac{1}{6} g_{o}(x)-\frac{1}{6} h_{o}(x)+\frac{1}{12} g_{e}(x)-\frac{1}{12} h_{e}(x) .
$$

So we obtain the following results.

Theorem 4.1. Let $\varphi: X^{2} \rightarrow[0, \infty)$ be a function such that there exists an $L<1$ with

$$
\varphi(x, y) \leq \frac{L}{16} \varphi(2 x, 2 y)
$$


for all $x, y \in X$. Let $f: X \rightarrow Y$ be a mapping satisfying $f(0)=0$ and (2.3). Then there exist an additive mapping $A: X \rightarrow Y$, a quadratic mapping $T: X \rightarrow Y$, a cubic mapping $C: X \rightarrow Y$ and $a$ quartic mapping $Q: X \rightarrow Y$ such that

$$
\begin{aligned}
\| f(x) & -\frac{1}{6} A(x)-\frac{1}{12} T(x)-\frac{1}{6} C(x)-\frac{1}{12} Q(x) \| \\
\leq & \left(\frac{L}{12-12 L}+\frac{L}{48-48 L}+\frac{L}{48-48 L}+\frac{L}{192-192 L}\right)(4 \varphi(x, x)+\varphi(2 x, x))
\end{aligned}
$$

for all $x \in X$.

Proof. Since $\varphi(x, y) \leq(L / 16) \varphi(2 x, 2 y), \varphi(x, y) \leq(L / 8) \varphi(2 x, 2 y), \varphi(x, y) \leq(L / 4) \varphi(2 x, 2 y)$ and $\varphi(x, y) \leq(L / 2) \varphi(2 x, 2 y)$. The result follows from Theorems 2.1, 2.5, 3.1, and 3.5.

Corollary 4.2. Let $\theta \geq 0$ and let $p$ be a real number with $p>4$. Let $f: X \rightarrow Y$ be a mapping satisfying $f(0)=0$ and (2.24). Then there exist an additive mapping $A: X \rightarrow Y$, a quadratic mapping $T: X \rightarrow Y$, a cubic mapping $C: X \rightarrow Y$ and a quartic mapping $Q: X \rightarrow Y$ such that

$$
\begin{aligned}
\| f(x) & -\frac{1}{6} A(x)-\frac{1}{12} T(x)-\frac{1}{6} C(x)-\frac{1}{12} Q(x) \| \\
\leq & \left(\frac{2^{p}+9}{6\left(2^{p}-2\right)}+\frac{2^{p}+9}{12\left(2^{p}-4\right)}+\frac{2^{p}+9}{6\left(2^{p}-8\right)}+\frac{2^{p}+9}{12\left(2^{p}-16\right)}\right) \theta\|x\|^{p}
\end{aligned}
$$

for all $x \in X$.

Theorem 4.3. Let $\varphi: X^{2} \rightarrow[0, \infty)$ be a function such that there exists an $L<1$ with

$$
\varphi(x, y) \leq 2 L \varphi\left(\frac{x}{2}, \frac{y}{2}\right)
$$

for all $x, y \in X$. Let $f: X \rightarrow Y$ be a mapping satisfying $f(0)=0$ and (2.3). Then there exist an additive mapping $A: X \rightarrow Y$, a quadratic mapping $T: X \rightarrow Y$, a cubic mapping $C: X \rightarrow Y$, and a quartic mapping $Q: X \rightarrow Y$ such that

$$
\begin{aligned}
\| f(x) & -\frac{1}{6} A(x)-\frac{1}{12} T(x)-\frac{1}{6} C(x)-\frac{1}{12} Q(x) \| \\
\leq & \left(\frac{1}{12-12 L}+\frac{1}{48-48 L}+\frac{1}{48-48 L}+\frac{1}{192-192 L}\right)(4 \varphi(x, x)+\varphi(2 x, x))
\end{aligned}
$$

for all $x \in X$.

Proof. Since $\varphi(x, y) \leq 2 L \varphi(x / 2, y / 2), \varphi(x, y) \leq 4 L \varphi(x / 2, y / 2), \varphi(x, y) \leq 8 L \varphi(x / 2, y / 2)$ and $\varphi(x, y) \leq 16 L \varphi(x / 2, y / 2)$. The result follows from Theorems 2.3, 2.7, 3.3, and 3.7. 
Corollary 4.4. Let $\theta \geq 0$ and let $p$ be a real number with $0<p<1$. Let $f: X \rightarrow Y$ be a mapping satisfying $f(0)=0$ and (2.24). Then there exist an additive mapping $A: X \rightarrow Y$, a quadratic mapping $T: X \rightarrow Y$, a cubic mapping $C: X \rightarrow Y$, and a quartic mapping $Q: X \rightarrow Y$ such that

$$
\begin{aligned}
\| f(x) & -\frac{1}{6} A(x)-\frac{1}{12} T(x)-\frac{1}{6} C(x)-\frac{1}{12} Q(x) \| \\
\leq & \left(\frac{2^{p}+9}{6\left(2-2^{p}\right)}+\frac{2^{p}+9}{12\left(4-2^{p}\right)}+\frac{2^{p}+9}{6\left(8-2^{p}\right)}+\frac{2^{p}+9}{12\left(16-2^{p}\right)}\right) \theta\|x\|^{p}
\end{aligned}
$$

for all $x \in X$.

\section{Acknowledgments}

The first and third authors were supported by Basic Science Research Program through the National Research Foundation of Korea funded by the Ministry of Education, Science and Technology (NRF-2009-0071229) and (NRF-2009-0070788), respectively.

\section{References}

[1] S. M. Ulam, A Collection of Mathematical Problems, Interscience Tracts in Pure and Applied Mathematics, no. 8, Interscience Publishers, New York, NY, USA, 1960.

[2] D. H. Hyers, "On the stability of the linear functional equation," Proceedings of the National Academy of Sciences of the United States of America, vol. 27, pp. 222-224, 1941.

[3] T. Aoki, "On the stability of the linear transformation in Banach spaces," Journal of the Mathematical Society of Japan, vol. 2, pp. 64-66, 1950.

[4] Th. M. Rassias, "On the stability of the linear mapping in Banach spaces," Proceedings of the American Mathematical Society, vol. 72, no. 2, pp. 297-300, 1978.

[5] P. Găvruţa, "A generalization of the Hyers-Ulam-Rassias stability of approximately additive mappings," Journal of Mathematical Analysis and Applications, vol. 184, no. 3, pp. 431-436, 1994.

[6] F. Skof, "Proprietà locali e approssimazione di operatori," Rendiconti del Seminario Matematico e Fisico di Milano, vol. 53, pp. 113-129, 1983.

[7] P. W. Cholewa, "Remarks on the stability of functional equations," Aequationes Mathematicae, vol. 27, no. 1-2, pp. 76-86, 1984.

[8] S. Czerwik, "On the stability of the quadratic mapping in normed spaces," Abhandlungen aus dem Mathematischen Seminar der Universität Hamburg, vol. 62, pp. 59-64, 1992.

[9] S. Czerwik, Functional Equations and Inequalities in Several Variables, World Scientific, River Edge, NJ, USA, 2002.

[10] G.-L. Forti, "Comments on the core of the direct method for proving Hyers-Ulam stability of functional equations," Journal of Mathematical Analysis and Applications, vol. 295, no. 1, pp. 127-133, 2004.

[11] G.-L. Forti, "Elementary remarks on Ulam-Hyers stability of linear functional equations," Journal of Mathematical Analysis and Applications, vol. 328, no. 1, pp. 109-118, 2007.

[12] D. H. Hyers, G. Isac, and Th. M. Rassias, Stability of Functional Equations in Several Variables, Progress in Nonlinear Differential Equations and Their Applications, 34, Birkhäuser, Boston, Mass, USA, 1998.

[13] S.-M. Jung, Hyers-Ulam-Rassias Stability of Functional Equations in Mathematical Analysis, Hadronic Press, Palm Harbor, Fla, USA, 2001.

[14] C. Park, "Hyers-Ulam-Rassias stability of a generalized Apollonius-Jensen type additive mapping and isomorphisms between $C^{*}$-algebras," Mathematische Nachrichten, vol. 281, no. 3, pp. 402-411, 2008.

[15] C. Park and A. Najati, "Homomorphisms and derivations in $C^{*}$-algebras," Abstract and Applied Analysis, vol. 2007, Article ID 80630, 12 pages, 2007. 
[16] J. M. Rassias and M. J. Rassias, "Asymptotic behavior of alternative Jensen and Jensen type functional equations," Bulletin des Sciences Mathématiques, vol. 129, no. 7, pp. 545-558, 2005.

[17] Th. M. Rassias, "On the stability of functional equations in Banach spaces," Journal of Mathematical Analysis and Applications, vol. 251, no. 1, pp. 264-284, 2000.

[18] Th. M. Rassias, "On the stability of functional equations and a problem of Ulam," Acta Applicandae Mathematicae, vol. 62, no. 1, pp. 23-130, 2000.

[19] Th. M. Rassias and P. Šemrl, "On the Hyers-Ulam stability of linear mappings," Journal of Mathematical Analysis and Applications, vol. 173, no. 2, pp. 325-338, 1993.

[20] K.-W. Jun and H.-M. Kim, "The generalized Hyers-Ulam-Rassias stability of a cubic functional equation," Journal of Mathematical Analysis and Applications, vol. 274, no. 2, pp. 267-278, 2002.

[21] S. H. Lee, S. M. Im, and I. S. Hwang, "Quartic functional equations," Journal of Mathematical Analysis and Applications, vol. 307, no. 2, pp. 387-394, 2005.

[22] J. K. Chung and P. K. Sahoo, "On the general solution of a quartic functional equation," Bulletin of the Korean Mathematical Society, vol. 40, no. 4, pp. 565-576, 2003.

[23] J. M. Rassias, "Solution of the Ulam stability problem for quartic mappings," Glasnik Matematički, vol. 34(54), no. 2, pp. 243-252, 1999.

[24] L. Cădariu and V. Radu, "Fixed points and the stability of Jensen's functional equation," Journal of Inequalities in Pure and Applied Mathematics , vol. 4, no. 1, article 4, 2003.

[25] J. B. Diaz and B. Margolis, "A fixed point theorem of the alternative, for contractions on a generalized complete metric space," Bulletin of the American Mathematical Society, vol. 74, pp. 305-309, 1968.

[26] G. Isac and Th. M. Rassias, "Stability of $\psi$-additive mappings: applications to nonlinear analysis," International Journal of Mathematics and Mathematical Sciences, vol. 19, no. 2, pp. 219-228, 1996.

[27] L. Cădariu and V. Radu, "On the stability of the Cauchy functional equation: a fixed point approach," in Iteration Theory (ECIT '02), vol. 346 of Grazer Math. Ber., pp. 43-52, Karl-Franzens-Univ. Graz, Graz, Austria, 2004.

[28] L. Cădariu and V. Radu, "Fixed point methods for the generalized stability of functional equations in a single variable," Fixed Point Theory and Applications, vol. 2008, Article ID 749392, 15 pages, 2008.

[29] M. Mirzavaziri and M. S. Moslehian, "A fixed point approach to stability of a quadratic equation," Bulletin of the Brazilian Mathematical Society, vol. 37, no. 3, pp. 361-376, 2006.

[30] C. Park, "Fixed points and Hyers-Ulam-Rassias stability of Cauchy-Jensen functional equations in Banach algebras," Fixed Point Theory and Applications, vol. 2007, Article ID 50175, 15 pages, 2007.

[31] C. Park, "Generalized Hyers-Ulam stability of quadratic functional equations: a fixed point approach," Fixed Point Theory and Applications, vol. 2008, Article ID 493751, 9 pages, 2008.

[32] V. Radu, "The fixed point alternative and the stability of functional equations," Fixed Point Theory, vol. 4, no. 1, pp. 91-96, 2003.

[33] D. Miheț and V. Radu, "On the stability of the additive Cauchy functional equation in random normed spaces," Journal of Mathematical Analysis and Applications, vol. 343, no. 1, pp. 567-572, 2008.

[34] M. Eshaghi-Gordji, S. Kaboli-Gharetapeh, C. Park, and S. Zolfaghri, "Stability of an additive-cubicquartic functional equation," Advances in Difference Euqations, vol. 2009, Article ID 395693, 20 pages, 2009.

[35] M. Eshaghi-Gordji, S. Abbaszadeh, and C. Park, "On the stability of a generalized quadratic and quartic type functional equation in quasi-Banach spaces," Journal of Inequalities and Applications, vol. 2009, Article ID 153084, 26 pages, 2009.

[36] M. Eshaghi-Gordji, S. Kaboli Gharetapeh, J. M. Rassias, and S. Zolfaghari, "Solution and stability of a mixed type additive, quadratic, and cubic functional equation," Advances in Difference Equations, vol. 2009, Article ID 826130, 17 pages, 2009.

[37] M. Eshaghi-Gordji, "Stability of a functional equation deriving from quartic and additive functions," preprint. 